\title{
Evidências de validade dos estilos de pensamento executivo, legislativo e judiciário
}

\author{
Cristiano Mauro Assis Gomes \\ Universidade Federal de Minas Gerais, Belo Horizonte-MG, Brasil \\ Eunaihara Ligia Lira Marques ${ }^{1}$ \\ Faculdade de Pará de Minas, Pará de Minas-MG, Brasil
}

\section{RESUMO}

Estudos têm indicado que os estilos de pensamento da teoria do autogoverno mental apresentam relações com o desempenho escolar. Não obstante sua relevância, apenas um estudo investigou diretamente a validade dos estilos de pensamento, em termos de estrutura fatorial de itens. O presente estudo teve o objetivo de investigar evidências de validade dos estilos de pensamento executivo, legislativo e judiciário. Participaram do estudo 278 alunos do ensino médio de uma escola pública do Estado de Minas Gerais, Brasil. Três testes de inteligência fluida da Bateria de Fatores Cognitivos de Alta-Ordem (BAFACALO), assim como o Inventário de Estilos de Pensamento (IEP - Grupo Funções) de Sternberg, foram aplicados. Foram utilizados procedimentos de análise fatorial confirmatória e modelamento por equação estrutural. Os resultados apontam evidências de validade favoráveis à estrutura fatorial dos três estilos de pensamento investigados e sua distinção diante das habilidades cognitivas. Implicações para a teoria são discutidas.

Palavras-chave: estilos de pensamento; inteligência; validade estatística; aptidão cognitiva.

\section{ABSTRACT - Validity evidence for the executive, legislative and judicial thinking styles}

Studies have showed that the thinking styles of the theory of mental self-government are related to academic achievement. Despite of their relevance, just one study directly investigated the validity of the thinking styles, in terms of items factor structure. The present study aims to investigate evidences of validity of the executive, legislative and judicial thinking styles. The sample was composed by 278 high school students of a public school in the State of Minas Gerais, Brazil. Three fluid intelligence tests from the Higher-Order Cognitive Factor Battery and the Sternberg' Thinking Styles Inventory (Functions) were administered. Confirmatory factor analysis and structural equation modeling were used. The results indicate favorable evidences about the factor structure of the three evaluated thinking styles and their differentiation on the cognitive abilities. Implications to the theory are discussed.

Keywords: thinking styles; intelligence; validity statistical; cognitive fitness.

\section{RESUMEN - Evidencias de validez de los estilos de pensamiento ejecutivo, legislativo y judicial}

Estudios han indicado que los estilos de pensamiento de la teoría del autogobierno mental presentan relaciones con el rendimiento escolar. Non obstante su relevancia, solamente un estudio ha investigado directamente la validez de los estilos de pensamiento, en términos de la estructura factorial de ítems. El presente estudio propone investigar las evidencias de validez de los estilos de pensamiento ejecutivo, legislativo y judicial. Participaran de lo estudio 278 alumnos de lo enseno medio de una escuela pública in el Estado de Minas Gerais, Brasil. Tres testes de inteligencia fluida de la Batería de Factores Cognitivos de Alta-Ordenen y lo Inventario de los Estilos de Pensamiento (Grupo de Funciones) de Sternberg fueran aplicados. Fueran utilizados procedimientos de análisis factorial confirmatoria y modelamiento por ecuación estructural. Los resultados indican evidencias favorables de la validez a respecto de la estructura factorial de los tres estilos de pensamiento investigados y su diferenciación en relación a ciertas habilidades cognitivas. Implicaciones para la teoría son discutidas.

Palabras clave: estilos de pensamiento; inteligencia; validez estadística; aptitud cognitivas.

Desde meados do século XX, a literatura sobre estilos tem atraído a atenção de pesquisadores em psicologia (Monreal, 2000). A definição de estilo é relativamente simples e psicologicamente atrativa. Um estilo é uma maneira preferencial que uma ou mais pessoas têm de pensar e agir diante de determinadas situações, mobilizando tanto processos cognitivos quanto emocionais (Monreal, 2000). Conceitualmente, uma característica fundamental dos estilos envolve sua distinção em relação às habilidades cognitivas. Os pesquisadores em estilos compreendem tais aptidões como "ferramentas mentais" ou "capacidades internas que permitem a ação", afirmando que os estilos são justamente as preferências de uso deste "ferramental interno" (as habilidades cognitivas) para uma determinada finalidade (Sternberg, 1994; Wechsler, 2006). 
Há uma variedade enorme de estilos propostos por um número também amplo e diverso de pesquisadores. Uma revisão realizada por Messick (1984) encontrou 8 classes de estilos cognitivos, enquanto um estudo feito por Hayes e Allinson (1994) identificou 29 classes de estilos. Inseridos nessa diversidade se situam os estilos de pensamento. Tendo sido criados na década de 1980 por Sternberg (1988), ao propor a teoria do autogoverno mental, os estilos de pensamento são caracterizados como as preferências das pessoas, o que está de acordo com a definição fundamental da literatura sobre estilos. Não obstante, os estilos de pensamento são preferências de pensamento, o que salienta o domínio desse tipo de estilo: o pensamento e as preferências de pensar (Sternberg \& Grigorenko, 1997a).

Sternberg (1988) faz uma analogia dos estilos de pensamento com dimensões do governo de uma sociedade e propõe a existência de 13 estilos agrupados em 5 dimensões de governança: funções, formas, níveis, tendências e âmbitos de governo. A dimensão das funções de governo - executar, criar e avaliar e/ou julgar - envolve os estilos de pensamento executivo, legislativo e judiciário. Por sua vez, os estilos de pensamento hierárquico, monárquico, oligárquico e anárquico correspondem à dimensão das formas de ação dos governos. Os estilos de pensamento global e local correspondem à dimensão dos níveis de administração central e regional. Os estilos de pensamento liberal e conservador compõem os grandes princípios e tendências orientadores, e os estilos de pensamento interno e externo correspondem aos âmbitos da soberania nacional e da ação integrada internacional.

$\mathrm{Na}$ medida em que cada estilo de pensamento determina uma preferência a respeito do modo de pensar, a teoria do autogoverno mental de Sternberg salienta a presença de 13 maneiras ou preferências distintas. Exemplificando a dimensão das funções, a teoria afirma que pessoas com predominância do estilo legislativo tendem a optar por criar, imaginar e improvisar nas situações do seu cotidiano, e geralmente estão preocupadas com a formulação de ideias, sugerindo uma maneira própria de fazer e decidir as coisas. Por sua vez, pessoas com predominância do estilo executivo tendem a gostar de cumprir regras bem definidas, pôr em prática as ideias de outros, ser direcionadas e seguir ordens em seu cotidiano e, ao resolver os problemas, frequentemente utilizam e cumprem regras pré-estruturadas ou preexistentes. Por fim, pessoas com predominância judiciária gostam de avaliar, comparar, criticar, julgar e analisar regras, procedimentos e ideias (Sternberg, 1988).

Do ponto de vista da relevância social da teoria do autogoverno mental, pode-se dizer que os estilos de pensamento são relevantes, pois eles "influenciam o processo de aprendizagem do discente e a dinâmica do ensino" (Gomes, Marques, \& Golino, 2014, p. 32). Estudos afirmam existir uma melhoria no desempenho dos estudantes quando as atividades acadêmicas se encontram em sintonia com os estilos de pensamento dos discentes e quando seus estilos correspondem aos estilos dos professores (Sternberg \& Grigorenko, 1997a).

Visando mensurar os 13 estilos de pensamento, Sternberg criou um instrumento de autorrelato: o Inventário de Estilos de Pensamento (IEP) (Sternberg, 1988). Na medida em que o IEP é o único instrumento voltado para a aferição dos estilos de pensamento, ele tem sido utilizado em todos os estudos empíricos sobre o tema. O inventário é composto de 13 escalas e 104 itens no total. Cada uma das escalas possui 8 itens e visa mensurar um dos 13 estilos de pensamento. Todos os itens do IEP possuem um enunciado que define uma preferência de pensar. Uma escala do tipo Likert, com o intervalo de 1 a 7 pontos, serve de referência para que as pessoas respondam a todos os itens do inventário.

Sternberg (1997) afirma que o IEP tem se mostrado válido e confiável para a mensuração dos 13 estilos. No entanto, tal afirmação parece ser excessivamente otimista quando confrontada com os resultados dos estudos que investigaram a estrutura fatorial do IEP e, por consequência, a identificação empírica dos estilos de pensamento ou das dimensões de governança.

Já em um de seus primeiros estudos a respeito dos estilos de pensamento, Sternberg (1988) se preocupou em analisar empiricamente a estrutura fatorial do IEP. Nesse estudo, ele investigou se os 13 escores das escalas do inventário eram capazes de indicar a presença empírica das 5 dimensões de governança (funções, formas, níveis, tendências e âmbitos). Ao realizar análises fatoriais exploratórias, inicialmente Sternberg (1988) esperava encontrar cinco fatores, cada um deles a ser carregado especificamente pelas escalas de uma determinada dimensão de governança. Por exemplo, um dos cinco fatores esperados deveria ser carregado pelas três escalas das funções de governança (estilos judiciário, legislativo e executivo); outro fator deveria ser carregado especificamente pelos estilos das formas de governança, ou seja, os estilos de pensamento hierárquico, monárquico, oligárquico e anárquico. Um terceiro fator deveria ser carregado unicamente pelos níveis de governança, ou seja, os estilos de pensamento global e local. Um quarto fator deveria ser carregado pelas tendências de governança, os estilos de pensamento liberal e conservador; e um quinto fator deveria ser carregado pelos estilos de pensamento interno e externo, que correspondem aos âmbitos de governança.

Sternberg (1988) identificou cinco fatores utilizando como critério de retenção de fatores o fundamento teórico. Em geral, os fatores identificados não corresponderam ao esperado pelos pressupostos teóricos. O primeiro fator foi carregado pelas escalas dos estilos legislativo, liberal, conservador e executivo, sendo que os estilos legislativo e liberal apresentaram cargas fatoriais positivas, enquanto os estilos conservador e executivo apresentaram cargas negativas; o segundo fator foi carregado pelos estilos judiciário e oligárquico; o terceiro fator foi carregado pelos 
estilos interno e externo; o quarto fator foi carregado pelos estilos global e local; e o quinto fator foi carregado exclusivamente pelo estilo hierárquico. Os estilos anárquico e monárquico não apresentaram cargas fatoriais estatisticamente significativas em nenhum dos fatores identificados.

A partir desse estudo seminal, investigações posteriores de Sternberg incorporaram a mesma estratégia de análise fatorial dos escores das 13 escalas, encontrando resultados bastante semelhantes aos do estudo original (Sternberg, 1994; Sternberg \& Grigorenko, 1997b). Além dos próprios estudos de Sternberg, outros pesquisadores realizaram investigações a respeito da estrutura fatorial do IEP, seguindo uma estratégia de análise de dados semelhante à empregada pelos estudos originais, de investigar a validade das cinco dimensões de governança (Dai \& Feldhusen, 1999; Fer, 2007; Zhang, 2002, 2006). Esses estudos trazem uma diversidade de amostras: estudantes de ensino médio, estudantes de ensino superior, estudantes superdotados e responsáveis de alunos do ensino médio. A Tabela 1 apresenta o número de fatores encontrados nesses estudos. Conforme pode ser observado, há divergências quanto ao número de fatores identificados. Ao mesmo tempo, pode-se perceber que vários fatores identificados tendem a caracterizar grupos híbridos, que possuem estilos de pensamento de mais de uma dimensão de governança (Tabela 1).

Os estudos mencionados são relevantes, mas nenhum deles avalia especificamente os estilos de pensamento. Todos avaliam a sustentação empírica das dimensões de governança da teoria. Apenas Black e McCoach (2008) investigam empiricamente a presença de 12 dos 13 estilos de pensamento, realizando uma série de análises fatoriais de itens. Os autores aplicaram o IEP a
789 estudantes do ensino médio ( $9^{\mathrm{a}}$ a $12^{\mathrm{a}}$ série) de 4 escolas americanas de Connecticut, Estados Unidos. Em função de problemas na aplicação da escala do estilo oligárquico, ela não pôde ser utilizada para a análise. Primeiramente, Black e McCoach (2008) investigaram a estrutura teórica das 5 dimensões de governança por meio da análise fatorial confirmatória dos escores das 12 escalas. O modelo testado determinava, a priori, a presença de cinco variáveis latentes (fatores) que representariam as dimensões de governança da teoria (funções, formas, níveis, tendências e âmbitos). Cada uma dessas variáveis latentes deveria explicar unicamente a variância dos escores das escalas teoricamente vinculadas. Por exemplo, as escalas das funções de governo, representando os estilos legislativo, judiciário e executivo, deveriam ser exclusivamente explicadas por uma variável latente (fator) que representaria a dimensão das funções de governança. Os resultados dessa análise apontaram para uma solução não convergente e os autores concluíram que a não convergência indicava que o modelo teórico das cinco dimensões de governança não se sustentava empiricamente.

Além do modelo teórico dos 5 agrupamentos, Black e McCoach (2008) analisaram modelos de 3, 2 e 4 fatores, também por meio de análises fatoriais confirmatórias dos escores das 12 escalas. Eles justificaram a relevância de testar esses modelos afirmando que essas soluções foram encontradas ou postuladas, respectivamente, nos estudos de Zhang e Sternberg (2005) — solução de três fatores - , Zhang (2002) — solução de dois fatores - e Zhang (2006) - solução de quatro fatores. Em síntese, os resultados indicaram que todos os modelos testados pela análise fatorial confirmatória ou não convergiram, apresentando problemas de estimação, ou apresentaram um ajuste inadequado aos dados.

Tabela 1

Estrutura fatorial e alfa de Cronbach relatados nos estudos

\begin{tabular}{|c|c|c|c|c|c|}
\hline Estudo & Fator 1 & Fator 2 & Fator 3 & Fator 4 & Fator 5 \\
\hline $\begin{array}{l}\text { Dai e Feldhusen } \\
\text { (1999) }\end{array}$ & $\begin{array}{c}\text { Legislativo, liberal, } \\
\text { anárquico, judiciário, } \\
\text { interno, local, hierárquico }\end{array}$ & $\begin{array}{c}\text { Executivo, local, } \\
\text { conservador, } \\
\text { monárquico, hierárquico }\end{array}$ & $\begin{array}{l}\text { Interno, } \\
\text { externo, } \\
\text { global }\end{array}$ & & \\
\hline Fer (2007) & $\begin{array}{l}\text { Oligárquico, } \\
\text { anárquico, local }\end{array}$ & $\begin{array}{l}\text { Legislativo, judiciário, } \\
\text { liberal, conservador }\end{array}$ & $\begin{array}{l}\text { Legislativo, } \\
\text { executivo, } \\
\text { hierárquico, }\end{array}$ & $\begin{array}{l}\text { Monárquico, } \\
\text { global }\end{array}$ & $\begin{array}{l}\text { Interno, } \\
\text { externo }\end{array}$ \\
\hline $\begin{array}{l}\text { Zhang e } \\
\text { Sternberg (2005) }\end{array}$ & $\begin{array}{l}\text { Legislativo, judiciário, } \\
\text { hierárquico, global, liberal }\end{array}$ & $\begin{array}{l}\text { Executivo, local, } \\
\text { monárquico, conservador }\end{array}$ & $\begin{array}{l}\text { Anárquico, } \\
\text { interno, } \\
\text { externo }\end{array}$ & & \\
\hline Zhang (2002) & $\begin{array}{l}\text { Legislativo, judiciário, } \\
\text { global, liberal }\end{array}$ & $\begin{array}{l}\text { Executivo, local, } \\
\text { conservador }\end{array}$ & & & \\
\hline Zhang (2006) & $\begin{array}{l}\text { Legislativo, liberal, } \\
\text { judiciário }\end{array}$ & $\begin{array}{c}\text { Executivo, conservador, } \\
\text { monárquico }\end{array}$ & Global, local & $\begin{array}{l}\text { Interno, } \\
\text { externo }\end{array}$ & \\
\hline
\end{tabular}

Nota: para apontar as relações entre os fatores e as escalas relacionadas a cada estilo, foi considerado como valor mínimo a carga de 0,40. O estudo de Zhang (2006) não informou as cargas fatoriais, de modo que todas as relações entre os fatores e as escalas foram informadas como no original. O estudo de Zhang (2002) utilizou apenas 7 das 13 escalas do Inventário de Estilos de Pensamento. Dai e Feldhusen (1999) aplicaram 12 das 13 escalas desse inventário. A pesquisa de Zhang e Sternberg (2005) não envolve nenhum estudo empírico, mas uma tentativa de Zhang e Sternberg de sintetizarem seus estudos anteriores e buscarem fatores consensuais, visando elaborar uma teoria integradora dos estilos, denominada de Threefold Model of Intellectual Styles. 
Em seguida, Black e McCoach (2008) realizaram uma análise fatorial confirmatória de itens. Todos os modelos testados determinavam que os 8 itens específicos de cada uma das 12 escalas deveriam ser explicados exclusivamente por 12 variáveis latentes (fatores) que representavam justamente os estilos de pensamento. Essa relação entre as 12 variáveis latentes (fatores) representando os estilos e seus itens-alvo representavam o primeiro nível de todos os modelos testados. A diferença entre os modelos se situava no segundo nível. Em um dos modelos testados, as 12 variáveis latentes representando os estilos eram explicadas por 5 variáveis latentes (fatores) de segunda ordem, que representavam as dimensões de governança da teoria; um segundo modelo testado determinava que as mesmas 12 variáveis latentes representando os estilos eram explicadas por 2 variáveis latentes de segundo nível; um terceiro modelo determinava a presença de 3 variáveis latentes de segundo nível; e um quarto modelo determinava a ocorrência de 4 variáveis latentes de segundo nível. Todos esses modelos correspondiam às soluções analisadas anteriormente pelas análises fatoriais confirmatórias dos escores das 12 escalas, sendo transpostos para a análise dos itens. Apenas o modelo de 12 fatores de primeiro nível e 2 fatores de segundo nível apresentou ajuste inadequado aos dados, enquanto todos os outros não convergiram.

Por último, Black e McCoach (2008) realizaram uma análise fatorial exploratória de itens, por meio do software SPSS 14.0, usando o principal axis factoring. Vários critérios foram utilizados para a retenção dos fatores: autovalores maiores do que 1 , teste scree, análise paralela, cargas iguais ou superiores a 0,35 para o fator mais importante de cada item, assim como o critério teórico. Os autores realizaram várias análises, eliminando fatores e inclusive itens que não eram carregados por nenhum dos fatores retidos. Concomitantemente, eles calculavam os coeficientes do alfa de Cronbach dos fatores retidos, e aqueles com valores inferiores a 0,70 eram eliminados. As soluções retidas foram rodadas com a rotação oblíqua oblimin. A solução final selecionou apenas 32 itens do IEP, identificando 5 fatores com um alfa de Cronbach variando entre 0,729 e 0,863 . Desses cinco fatores, apenas três representavam estilos preconizados pela teoria. O primeiro fator foi carregado por sete itens da escala do estilo liberal e dois itens da escala do estilo legislativo. O segundo fator foi carregado exclusivamente por itens da escala do estilo externo. Por sua vez, o terceiro fator foi carregado por seis itens da escala do estilo hierárquico, enquanto o fator quatro, por cinco itens da escala do estilo judiciário, e o quinto fator, por cinco itens da escala do estilo legislativo e um item da escala do estilo interno.

Além dos estudos que investigaram a identificação empírica dos estilos de pensamento e das dimensões de governança, podemos citar um estudo, diferente dos estudos originais de Sternberg, que visou analisar empiricamente o pressuposto teórico de que as habilidades cognitivas e os estilos são construtos distintos. Zhang (2004) aplicou o teste de habilidades triárquicas (Sternberg, 1993) a 250 alunos do ensino fundamental, com o IEP, e analisou as correlações entre os estilos e as inteligências prática, analítica e criativa da teoria triárquica da inteligência de sucesso de Sternberg (1993). Ela obteve resultados consistentes com aqueles encontrados por Sternberg e Grigorenko (1997a), de que as habilidades cognitivas e os estilos são construtos distintos. As escalas do teste de habilidades triárquicas apresentaram alfa de 0,60 na habilidade analítica, 0,66 na habilidade criativa e 0,34 na habilidade prática. Por apresentar alfa muito baixo, a última foi desconsiderada. As 13 escalas dos estilos de pensamento mostraram um alfa que variou entre 0,50 e 0,80 . A respeito das relações entre os estilos e as habilidades, houve apenas 2 correlações estatisticamente significativas, embora fracas, entre o estilo local e a habilidade analítica $(0,15)$, e entre o estilo anárquico e a habilidade criativa $(0,18)$.

Sintetizando a respeito da literatura sobre a estrutura fatorial dos estilos de pensamento, das dimensões de governança e de sua diferenciação em relação às habilidades cognitivas, pode-se afirmar que os estudos se concentraram entre os anos 1980 e 2000, sugerindo maior preocupação com a validade interna e externa dos estilos de pensamento nesse período de tempo. Quanto à literatura brasileira, não foram encontradas referências de estudos sobre os estilos de pensamento da teoria do autogoverno mental de Sternberg (1988). Considerando que a própria literatura internacional carece de estudos que investigam com mais profundidade e abrangência a estrutura fatorial dos estilos de pensamento, pois, até o momento, apenas um estudo alcançou esse objetivo, encontrando resultados desfavoráveis à teoria, o presente estudo teve o objetivo de realizar uma análise fatorial de itens para investigar a validade dos estilos de pensamento. Concomitantemente, o presente estudo também pretendeu investigar se os estilos de pensamento se distinguem de certas habilidades cognitivas. Este estudo não tencionou investigar a estrutura fatorial de todos os 13 estilos de pensamento da teoria. Especificamente, serão analisados os estilos executivo, legislativo e judiciário, ou seja, as funções de governança, em termos de sua estrutura fatorial e sua distinção diante de certas habilidades cognitivas.

\section{Método}

\section{Participantes}

A amostra que compôs o estudo foi de conveniência. Fizeram parte deste estudo 278 alunos do ensino médio de uma escola da rede pública do Estado de Minas Gerais, com idade entre 14 e 42 anos, com média de 16,94 e desvio padrão de 3,37 anos. Desse total, 93 (33,5\%) eram alunos do $1^{\circ}$ ano, $58(20,9 \%)$, alunos do $2^{\circ}$ ano, $102(36,7 \%)$, alunos do $3^{\circ}$ ano, e 25 (9\%), alunos da educação de jovens e adultos (EJA). Do total da amostra, 196 (70,5\%) participantes eram do sexo feminino, e $82(29,5 \%)$, do sexo masculino. 


\section{Instrumentos}

Inventário dos Estilos de Pensamento (Grupo das Funções) - versão traduzida

e adaptada para o português brasileiro

O IEP original foi elaborado, em inglês, por Sternberg (1997). Em função disso, seus itens que mensuram os três estilos de pensamento das funções da teoria do autogoverno (estilos executivo, legislativo e judiciário) foram traduzidos e adaptados pelos autores deste artigo, tendo em vista os objetivos do estudo. Cada um dos autores produziu uma tradução. As diferentes traduções foram comparadas, até a elaboração de uma tradução consensual e única. Em suma, todos os processos envolvidos com a tradução foram conduzidos exclusivamente pelos autores deste artigo, que não são tradutores profissionais ou bilíngues. A tradução foi caracterizada pelo processo concomitante de adaptação de termos específicos, expressões de linguagem próprias do inglês e outros aspectos contextuais. Por exemplo, o item de número 3, originalmente "I like to play with $m y$ ideas and see how far they go", poderia ser traduzido por "Eu gosto de brincar com minhas ideias e ver até onde elas vão". No entanto, o enunciado foi adaptado para "Eu gosto de gerar ideias e ver o que eu consigo alcançar com elas". Um estudo piloto com pessoas de diferentes níveis educacionais foi realizado visando verificar a pertinência das adaptações e o entendimento das pessoas em relação aos enunciados.

O IEP (Grupo das Funções) mede unicamente os três estilos de pensamento da dimensão das funções de governança. Cada um dos 3 estilos é medido por 8 itens específicos, de forma que o inventário grupo das funções é composto por 24 itens. Cada item possui um enunciado que expressa uma maneira específica de pensar, representando o estilo legislativo, ou o estilo executivo, ou o estilo judiciário. A resposta a cada item é avaliada por meio de uma escala do tipo Likert, que corresponde ao quanto o enunciado se refere à preferência pessoal do respondente (o valor 1 indica nenhuma relação, enquanto o valor 7 indica uma relação intensa).

Conjunto de testes de inteligência fluida, versão resumida, da Bateria de Fatores Cognitivos de Alta-Ordem

Elaborado por Gomes (2010), é formado por três testes capazes de mensurar as habilidades de raciocínio lógico, raciocínio geral e indução, assim como, em nível mais abrangente, a inteligência fluida (Gomes, 2010; Gomes \& Borges, 2009). Nesta versão resumida, o teste de indução (I) é composto por oito itens. Cada um dos seus itens é formado por cinco grupos de quatro letras. Desses, quatro grupos apresentam um mesmo padrão, uma mesma regra de organização de suas letras. $\mathrm{O}$ respondente deve identificar o grupo que não apresenta esse padrão e marcá-lo com um x. Na versão resumida, o tempo limite para sua realização é de seis minutos. A versão resumida do teste de raciocínio lógico (RL) é composta por 11 itens. Cada item do teste possui uma conclusão proveniente de duas premissas lógicas que não têm nenhuma relação empírica com o mundo. O objetivo do respondente é indicar se a conclusão presente no item é adequada ou inadequada em relação às suas premissas, marcando uma de duas opções dadas, com um tempo limite de oito minutos para a versão resumida. Já o teste de raciocínio geral (RG), versão resumida, possui oito itens que apresentam problemas lógico-matemáticos. $\mathrm{O}$ respondente deve interpretar o enunciado, resolver o problema e escolher 1 de 5 opções de respostas do conjunto de múltipla escolha, em um tempo limite de 12 minutos.

Gomes e Borges (2009) afirmam que esses testes apresentam unidimensionalidade, validade convergente e validade discriminante. Este estudo aplicou os testes do conjunto a 558 estudantes dos ensinos fundamental e médio (50,2\% do sexo feminino e $49,8 \%$ do sexo masculino) e verificou que o modelo bifatorial de itens representa adequadamente o desempenho dos participantes em cada um dos testes, sendo que o fator geral é o que melhor explica a variância em cada um dos testes. Gomes e Borges (2009) também verificaram que os três testes do conjunto são explicados especificamente por um fator (inteligência fluida), enquanto três testes de fluência são explicados por outro fator (fluência). Gomes (2010) obteve resultados provenientes de uma segunda análise que apontou os testes do conjunto como marcadores da inteligência fluida. Ele investigou os 3 testes do conjunto com outros 15 testes de inteligência e, teoricamente, marcadores de outras habilidades cognitivas. Todos os 18 testes avaliados pertencem à BAFACALO. Gomes (2010) realizou análises fatoriais exploratórias e confirmatórias dos escores dos 18 testes em uma amostra de 292 estudantes do ensino médio. Os resultados desse estudo apresentaram evidências favoráveis de que os 18 testes da bateria eram explicados por suas habilidades cognitivas-alvo. E, dentre eles, os três testes do conjunto de testes de inteligência fluida eram explicados pela habilidade cognitiva de inteligência fluida. Todos os testes avaliados apresentaram alfa de Cronbach superior a 0,70 (Gomes, 2010; Gomes \& Borges, 2009).

\section{Procedimentos de coleta e análise de dados}

A coleta dos dados ocorreu na própria escola. Os testes foram aplicados conjuntamente e de forma coletiva por um dos autores deste estudo em 20 turmas escolares, sendo 2 sessões por turma, com tempo médio de 50 minutos por sessão. Na primeira sessão, os participantes responderam aos itens do IEP, assim como ao teste de indução, e na segunda sessão, aos testes de raciocínio lógico e raciocínio geral. Foram respeitados todos os aspectos éticos necessários em relação aos participantes, sob a aprovação de comitê de ética, número de registro ETIC 463/11, da Universidade Federal de Minas Gerais (UFMG). 
Para analisar a estrutura fatorial do IEP (Grupo Funções), realizou-se uma análise fatorial confirmatória de itens, visando investigar um modelo que determinava a presença de três variáveis latentes (legislativo, executivo e judiciário) que explicavam os respectivos itens-alvo de cada um dos estilos da dimensão das funções de governança. Para investigar a diferenciação dos estilos de pensamento em relação às habilidades cognitivas, foi realizada uma análise por meio do modelamento por equação estrutural de itens, buscando verificar as relações entre os três estilos de pensamento e as habilidades cognitivas de raciocínio geral, raciocínio lógico e indução, mensuradas pelo conjunto de testes de inteligência fluida da BAFACALO (Gomes, 2010; Gomes \& Borges, 2009). O software utilizado foi o Mplus, versão 5.2, por meio da estimativa dos quadrados últimos ponderados robustos - robust weighted least squares estimator (WLSMV) - empregada em casos de escores de escalas do tipo Likert.

O ajuste dos modelos aos dados foi avaliado, neste trabalho, por meio do Índice Comparativo de Ajuste Comparative Fit Index (CFI) - e do Erro de Aproximação da Raiz Quadrada Média - Root Mean Square Error of Approximation (RMSEA). Valores iguais ou superiores a 0,95 no CFI e inferiores a 0,06 no RMSEA indicam um bom ajuste do modelo aos dados. Por sua vez, valores abaixo a 0,90 no CFI e iguais ou acima de 0,10 no RMSEA indicam soluções inadequadas, com pobre ajuste aos dados (Schumacker \& Lomax, 2004).

\section{Resultados e Discussão}

O primeiro resultado a ser descrito envolve a análise da relação teórica dos estilos executivo, judiciário e legislativo e seus respectivos itens marcadores do IEP (Grupo das Funções). O modelo testado foi composto por:

1. três variáveis latentes representando os três estilos avaliados;

2. 24 variáveis observáveis, representando as respostas das pessoas aos 24 itens do IEP (Grupo das Funções); e

3. variáveis latentes representando os erros ou resíduos das variáveis observáveis (itens) e das variáveis latentes representando os estilos.

Esse modelo determinava que cada estilo (variável latente) poderia carregar fatorialmente apenas os seus itens-alvo. Assim, cada estilo explicava a variância de oito itens. Para os itens não relacionados teoricamente a um estilo, o modelo fixava previamente uma carga fatorial (beta) de valor zero. Foi permitido que as variáveis latentes representantes dos três estilos se correlacionassem. Esse primeiro modelo gerou um ajuste insatisfatório por meio do indicador CFI, abaixo de $0,90\left(\chi^{2}[249]=626,33\right.$; $\mathrm{CFI}=0,88$; RMSEA $=0,07$ ).

Uma análise dos índices de modificação foi realizada visando verificar se o ajuste da solução melhoraria significativamente se alguns itens pudessem ser explicados também por outros estilos, além do estilo vinculado teoricamente ao item. O índice de modificação permite essa análise, pois informa o quanto uma solução é melhorada em função do acréscimo de uma nova relação ao modelo Tal verificação mostrou que o ajuste obteve sucesso. Em vez de modelar esse acréscimo, os itens que demandavam essa condição (de números 4, 8, 15 a 18 e 21) foram eliminados. Desses, 2 se relacionavam teoricamente ao estilo legislativo (itens 4 e 8), 2 estavam vinculados ao estilo judiciário (itens 15 e 16) e 3 eram itens marcadores do estilo executivo (itens 17, 18 e 21). Um novo modelo, já com esses itens excluídos, foi testado.

A nova solução apresentou adequado grau de ajuste aos dados $\left(\chi^{2}[116]=220,43 ; \mathrm{CFI}=0,94 ; \mathrm{RMSEA}=0,05\right.$; RMSEA [intervalo de confiança de $90 \%$ ] IC $90 \%$ 0,04-0,07), sustentando evidências favoráveis à validade dos estilos de pensamento executivo, legislativo e judiciário. A Tabela 2 apresenta as relações entre os itens e as variáveis latentes desse modelo. Todos os itens apresentaram beta maior ou igual a 0,42 , indicando que os itens selecionados são bem explicados pelo seu estilo-alvo. As cargas (betas) de valor zero foram omitidas na Tabela 2. Pode ser observado que as omissões ocorrem especificamente entre os itens e os estilos que não são o seu estilo-alvo. Os coeficientes do alfa de Cronbach dos itens selecionados foram de 0,68 para o estilo legislativo, 0,74 para o estilo executivo e 0,76 para

Tabela 2

Estrutura fatorial do Inventário de Estilos de Pensamento

\begin{tabular}{lll}
\hline \multicolumn{3}{c}{ Itens } \\
\hline i1 & 0,42 & \\
i3 & 0,57 & \\
i5 & 0,54 & \\
i6 & 0,54 & \\
i7 & 0,66 & \\
i9 & 0,58 & \\
i10 & 0,47 & \\
i11 & 0,65 & \\
i12 & 0,64 & \\
i13 & 0,70 & \\
i14 & 0,64 & \\
i19 & 0,67 & \\
i20 & 0,59 & \\
i22 & 0,68 & \\
i23 & 0,71 & \\
i24 & 0,73 & \\
\hline
\end{tabular}

Nota: i=item; todos os betas apresentaram um valor de $\mathrm{p} \leq 0,0009$. 
o estilo judiciário. Os 3 estilos apresentaram correlações moderadas entre si $(0,58$ entre os estilos judiciário e executivo, 0,54 entre os estilos judiciário e legislativo e 0,45 entre os estilos executivo e legislativo), indicando a provável presença de um fator geral.

Além da análise da estrutura fatorial do IEP (Grupo Funções) e da plausibilidade empírica dos estilos judiciário, executivo e legislativo, enquanto variáveis latentes capazes de explicar as respostas das pessoas aos itens marcadores desses estilos, este estudo buscou investigar a diferenciação dos estilos de pensamento em relação às habilidades cognitivas. Para isso, foi testado um modelo que determinava que os estilos de pensamento executivo, judiciário e legislativo seriam representados por três variáveis latentes e que essas explicariam a variância dos seus respectivos itens-alvo, conforme a solução já apresentada e após adequado ajuste aos dados. Além da parte relacionada aos estilos de pensamento, o modelo incluiu três novas variáveis latentes, tendo a função de representar as habilidades cognitivas de raciocínio geral, raciocínio lógico e indução. Esse modelo determinava que apenas a variável latente de raciocínio geral explicava a variância dos itens do teste de raciocínio geral, assim como apenas a variável latente de raciocínio lógico explicava a variância dos itens do teste de raciocínio lógico e apenas a variável latente de indução explicava a variância dos itens do teste de indução. Foi verificado que 2 dos 8 itens do teste de raciocínio geral e do 4 dos 11 itens do teste de raciocínio lógico estavam diminuindo o valor do alfa de Cronbach, sendo, por esse motivo, eliminados da análise. Nenhum item do teste de indução foi eliminado, perfazendo um total de 6 itens eliminados do total de 27 . Os resultados indicaram alfa de 0,60 para raciocínio geral, de 0,73 para raciocínio lógico e de 0,76 para indução.

O modelo apresentou adequado grau de ajuste $\left(\chi^{2}[650]=830,33 ; \mathrm{CFI}=0,94\right.$; RMSEA $=0,03$; RMSEA IC90\% 0,03-0,04), indicando evidências favoráveis à diferenciação dos estilos judiciário, executivo e legislativo e das habilidades cognitivas de raciocínio geral, raciocínio lógico e indução. A Tabela 3 apresenta os betas de cada variável latente em relação a todos os itens do modelo testado, englobando tanto os itens dos três estilos quanto os itens dos três testes de inteligência. Pode-se observar, na Tabela 3, que todas as seis variáveis latentes carregam fatorialmente apenas os seus itens-alvo. Por exemplo, o estilo legislativo apenas explica a variância das respostas das pessoas nos itens relacionados teoricamente a ele. Os espaços vazios na Tabela 3 indicam a presença de uma carga fatorial (beta) de valor zero.

Em suma, a presença dos betas apenas nos itens-alvo sustenta evidências de que os estilos se diferenciam claramente tanto uns dos outros como das habilidades cognitivas. Quanto à relação entre as variáveis latentes e seus itens-alvo, os betas apresentam valores iguais ou superiores a 0,30 , indicando que os itens estão
Tabela 3

Estrutural fatorial do Inventário de Estilos de Pensamento, Raciocínio Geral, Raciocínio Lógico e Indução

\begin{tabular}{|c|c|c|}
\hline & Itens & \\
\hline i 1 & 0,38 & \multirow{6}{*}{ Estilo legislativo } \\
\hline i 2 & 0,54 & \\
\hline i 3 & 0,52 & \\
\hline i 5 & 0,54 & \\
\hline i 6 & 0,70 & \\
\hline i 7 & 0,61 & \\
\hline i 9 & 0,46 & \multirow{6}{*}{ Estilo judiciário } \\
\hline i 10 & 0,63 & \\
\hline i 11 & 0,65 & \\
\hline i 12 & 0,70 & \\
\hline i 13 & 0,66 & \\
\hline i 14 & 0,66 & \\
\hline i 19 & 0,61 & \multirow{5}{*}{ Estilo executivo } \\
\hline i 20 & 0,67 & \\
\hline i 22 & 0,71 & \\
\hline i 23 & 0,72 & \\
\hline i 24 & 0,57 & \\
\hline RG 1 & 0,55 & \multirow{6}{*}{ RG } \\
\hline RG 3 & 0,82 & \\
\hline RG 8 & 0,75 & \\
\hline RG 9 & 0,61 & \\
\hline RG 11 & 0,40 & \\
\hline RG 15 & 0,23 & \\
\hline RL 1 & 0,80 & \multirow{7}{*}{$\mathrm{RL}$} \\
\hline RL 2 & 0,87 & \\
\hline RL 3 & 0,78 & \\
\hline RL 6 & 0,59 & \\
\hline RL 16 & 0,77 & \\
\hline RL 23 & 0,61 & \\
\hline RL 29 & 0,47 & \\
\hline I 1 & 0,79 & \multirow{8}{*}{ I } \\
\hline I 4 & 0,74 & \\
\hline I 5 & 0,84 & \\
\hline I 6 & 0,80 & \\
\hline I 9 & 0,72 & \\
\hline I 10 & 0,36 & \\
\hline I 14 & 0,36 & \\
\hline I 15 & 0,48 & \\
\hline
\end{tabular}

Nota: i=item do Inventário de Estilos de Pensamento (Grupo Funções); RG=raciocínio geral; RL=raciocínio lógico; I=indução; todos os betas apresentaram um valor de $p \leq 0,0009$, com exceção de I15, que apresentou um $p=0,01$. 
minimamente bem explicados pelas variáveis latentes. A única exceção é o item RG15, que apresenta um beta de 0,23 . Focando nos estilos, por serem o alvo do presente estudo, o estilo legislativo apresentou betas que variaram de 0,38 a 0,70 em seus itens-alvo; os betas do estilo judiciário variaram de 0,46 a 0,70 ; já o estilo executivo teve betas de 0,57 a 0,72 . Todos esses betas indicam que os estilos de pensamento explicam, pelo menos razoavelmente, seus itens-alvo.

Quanto às relações entre as variáveis latentes do modelo testado, os três estilos apresentam correlações moderadas entre si. O mesmo ocorre entre as três habilidades cognitivas, que apresentam correlações moderadas a altas entre si. Por sua vez, as correlações de cada um dos estilos com as habilidades cognitivas apresentam valores fracos (Tabela 4). Essa condição é uma evidência favorável também à diferenciação dos estilos de pensamento em relação às habilidades cognitivas.

Pode-se concluir que a maior associação entre as variáveis latentes que compõem o grupo dos estilos de pensamento e a maior associação entre as variáveis latentes que compõem o grupo das habilidades cognitivas reforçam a evidência favorável a respeito da diferenciação dos estilos de pensamento em relação às habilidades cognitivas. Tal evidência, que foi inicialmente construída a partir da análise da relação entre as variáveis latentes e os itens do modelo (Tabela 3), é ampliada, pois, pela análise das correlações das variáveis latentes (Tabela 4).

Ponderando sobre o grau de ajuste do modelo avaliado pela análise fatorial confirmatória de itens e do modelo avaliado pelo modelamento por equação estrutural de itens, apesar de Hu e Bentler (1999) sugerirem que o CFI $\geq 0,95$ seja um critério mais exato de um bom ajuste do modelo aos dados, os modelos testados apresentaram CFI próximo a esse valor, mostrando não um bom, mas razoável ajuste. No entanto, essa condição do ajuste aos dados deve ser relativizada, visto que os modelos elaborados foram bastante rigorosos no sentido de permitir que as variáveis latentes explicassem exclusivamente os seus itens-alvo. É muito provável que se os modelos permitissem algumas relações entre as variáveis latentes e outros itens que não seus itens-alvo, o valor do CFI aumentaria e atingiria o critério de bom ajuste. Por outro lado, devese ressaltar que todos os valores do índice RMSEA dos modelos foram iguais ou menores a 0,05 , indicando bom ajuste aos dados.

O resultado médio do alfa de Cronbach para os 3 estilos de pensamento foi de 0,72 . Esse valor médio é similar aos valores relatados em investigações anteriores sobre os alfas dos estilos judiciário, legislativo e executivo (Black \& McCoach, 2008; Dai \& Feldhusen, 1999; Fer, 2007; Sternberg, 1988, 1994; Zhang, 2002), apesar de essa afirmação de similaridade dos alfas não se assentar em nenhum tratamento estatístico.

A partir da sustentação empírica das três variáveis latentes que representam os estilos executivo, legislativo e judiciário, é possível afirmar favoravelmente a sua existência, como proposto pela teoria do autogoverno mental de Sternberg (1988), e que os estilos de pensamento se diferenciam de habilidades cognitivas de raciocínio, o que corrobora resultados de investigações anteriores (Sternberg \& Grigorenko, 1997b; Zhang, 2004). No entanto, é relevante informar que os estudos anteriores compararam os estilos com habilidades cognitivas da teoria de inteligência de sucesso de Sternberg (1985). Já o presente estudo comparou os estilos com habilidades cognitivas de uma bateria de testes de inteligência baseada no modelo Cattell-Horn-Carroll (CHC), considerado o estado-da-arte dos modelos sobre inteligência humana.

Ainda no que tange às novas informações obtidas neste estudo, se os resultados das fracas correlações dos estilos em relação às habilidades cognitivas, apresentadas na Tabela 4, são informações semelhantes aos resultados encontrados nos estudos anteriores, as relações exclusivas entre as variáveis latentes (estilos e habilidades) e seus itens-alvo, com betas de valor zero para todos os outros itens (Tabela 3), são informações que ainda não tinham sido obtidas pela literatura da área.

Tabela 4

Matriz de correlação das variáveis latentes

\begin{tabular}{lccccc}
\hline & $\begin{array}{c}\text { Estilo } \\
\text { legislativo }\end{array}$ & $\begin{array}{c}\text { Estilo } \\
\text { judiciário }\end{array}$ & $\begin{array}{c}\text { Estilo } \\
\text { executivo }\end{array}$ & $\begin{array}{c}\text { Raciocínio } \\
\text { geral }\end{array}$ & $\begin{array}{c}\text { Raciocínio } \\
\text { lógico }\end{array}$ \\
\hline Estilo legislativo & 1,00 & & & & \\
Estilo judiciário & $0,54^{*}$ & 1,00 & & & \\
Estilo executivo & $0,45^{*}$ & $0,58^{*}$ & 1,00 & 1,00 & 1,00 \\
Raciocínio geral & $0,23^{*}$ & 0,13 & 0,13 & $0,73^{*}$ & $0,69^{*}$ \\
Raciocínio lógico & $0,18^{* *}$ & 0,12 & 0,15 & $0,68^{*}$ & 1,00 \\
Indução & 0,06 & $-0,02$ & 0,10 & & \\
\hline
\end{tabular}

Nota: ${ }^{*} p \leq 0,01 ;{ }^{* *} p \leq 0,05$. 
Conforme exposto, a sustentação empírica dos 13 estilos ainda é um desafio em aberto, e as evidências existentes não são favoráveis para a identificação de todos os estilos propostos. Apesar de uma série de pesquisas ter investigado a estrutura fatorial do IEP, todas elas, com exceção de Black e McCoach (2008), investigaram a validade das dimensões de governança, e não a validade da estrutura fatorial dos estilos. Nesse sentido, o presente estudo tem apenas o estudo de Black e McCoach (2008) como referência para comparação de seus resultados: este estudo teve um escopo consideravelmente mais reduzido (24 itens referentes de 3 escalas do IEP) do que o estudo de Black e McCoach (2008)(96 itens de 12 escalas do IEP). No entanto, o presente estudo manteve 17 itens dos 24 analisados, por uma escolha de eliminação dos itens que demandavam que mais de um fator explicasse sua variância. Comparando as eliminações de itens, conclui-se que o presente estudo gerou uma exclusão menos impactante do que o estudo de Black e McCoach (2008). Além disso, os modelos testados apresentaram razoável grau de ajuste aos dados, enquanto os modelos de Black e McCoach (2008) ou apresentaram problemas de convergência, ou convergiram, mas mostraram inadequado ajuste aos dados.

Como de praxe para todo estudo que possui uma amostra restrita e não representativa de uma determinada população, as conclusões aqui obtidas devem ser consideradas com cautela. Estudos posteriores com novas amostras são necessários para que as conclusões geradas ganhem um caráter mais generalizável e robusto.

Apesar da relevância dos resultados produzidos, talvez a maior contribuição da presente pesquisa seja salientar a importância de novos estudos sobre a estrutura fatorial de itens como forma de escrutínio da validade dos estilos de pensamento. Este estudo utilizou uma estratégia de análise diferente da comumente adotada pela literatura internacional da área. Esta tem tradicionalmente partido do princípio de que os itens relacionados em teoria com os estilos são adequados. No geral, a literatura da área tem investigado empiricamente as dimensões de governança, ao realizar análises fatoriais dos escores das escalas do IEP. De fato, a análise das dimensões é relevante e importante, mas sua ocorrência não poderia ou deveria eliminar a análise dos itens e, por consequência, a análise da sustentação empírica dos estilos de pensamento. O presente estudo salienta a necessidade de que sejam feitos muitos novos estudos sobre análise fatorial de itens dos estilos de pensamento. Esse tipo de análise permitirá que a proposição teórica dos 13 estilos ganhe maior robustez empírica. A existência de apenas dois estudos sobre o tema indica uma carência que deve ser trabalhada pelos pesquisadores interessados nesse interessante e promissor domínio de conhecimento.

\section{Referências}

Black, A. C., \& McCoach, D. B. (2008). Validity study of the Thinking Styles Inventory. Journal for the Education of the Gifted, 32(2), 180-210. Recuperado de http://files.eric.ed.gov/fulltext/EJ826290.pdf

Dai, D. Y., \& Feldhusen, J. F. (1999). A validation study of the Thinking Styles Inventory: Implications for gifted education. Roeper Review, 21(4), 302-307. doi: 10.1080/02783199909553981

Fer, S. (2007). What are the thinking styles of Turkish student teachers? Teachers College Record, 109(6), 1488-1516.

Gomes, C. M. A. (2010). Estrutura fatorial da Bateria de Fatores Cognitivos de Alta-Ordem (BaFaCAlO). Avaliação Psicológica, 9(3), 449-459. Recuperado de http://pepsic.bvsalud.org/scielo.php?script=sci_arttext\&pid=S1677-04712010000300011

Gomes, C. M. A., \& Borges O. N. (2009). Qualidades psicométricas do conjunto de testes de inteligência fluida. Avaliação Psicológica, 8(1), 17-32. Recuperado de http://pepsic.bvsalud.org/pdf/avp/v8n1/v8n1a03.pdf

Gomes, C. M. A., Marques, E. L. L., \& Golino, H. F. (2014). Validade incremental dos estilos legislativo, executivo e judiciário em relação ao rendimento escolar. Revista E-PSI, 3, 31-46. Recuperado de http://www.revistaepsi.com

Hayes, J., \& Allinson C. W. (1994). Cognitive style and its relevant for management practice. British Journal of Management, 5(1), 53-71. doi: 10.1111/j.1467-8551.1994.tb00068.x

Hu, L. T., \& Bentler, P. M. (1999). Cutoff criteria for fit indexes in covariance structure analysis: Conventional criteria versus new alternatives. Structural Equation Modeling, 6(1), 1-55. doi: 10.1080/10705519909540118

Messick, S. (1984). The nature of cognitive style: Problems and promise in educational practice. Educational Psychologist, 19(2), 59-74. doi: 10.1080/00461528409529283

Monreal, C. A. (2000). ¿Qué es la creatividad? Madrid: Biblioteca Nueva. Recuperado de http://www.scielo.org.ar/scielo.php?script=sci_ arttext\&pid $=$ S1668-81042008000200001

Schumacker, R. E., \& Lomax, R.G. A beginner's guide to structural equation modeling. ${ }^{\text {nd }}$ Edition Lawrence Erlbaum Associates, Inc.; Mahwah, New Jersey: 2004.

Sternberg, R. J. (1985). Beyond IQ: A triarchic theory of human intelligence. New York: Cambridge University Press.

Sternberg, R. J. (1988). Mental self-government: A theory of intellectual styles and their development. Human Development, 31(4), $197-224$. doi:10.1159/000275810 
Sternberg, R. J. (1993). Triarchic abilities test (Level H). (Teste não publicado). New Haven: Yale University.

Sternberg, R. J. (1994). Thinking styles: Theory and assessment at the interface between intelligence and personality. Em R. J. Sternberg \& P. Ruzgis (Eds.), Intelligence and personality. (pp. 169-187). New York: Cambridge University Press.

Sternberg, R. J. (1997). Thinking styles. Cambridge: Cambridge University Press.

Sternberg, R. J., \& Grigorenko, E. H. (1997a). Styles of thinking, abilities, and academic performance. Exceptional Children, 63(3), $295-312$. Recuperado de http:/www.freepatentsonline.com/article/Exceptional-Children/19200718.html. doi: 10.1177/001440299706300301

Sternberg, R. J., \& Grigorenko, E. H (1997b). Are cognitive styles still in style? American Psychologist, 52(7), 700-712. Recuperado de http:// www.beteronderwijsnederland.nl/files/sternberg\%20grigrenko.pdf. doi: 10.1037/0003-066X.52.7.700

Wechsler, S. M. (2006). Estilo de pensar e criar. Campinas: IDB/LAMP-PUC- Campinas.

Zhang, L. F. (2002). Thinking styles: Their relationships with modes of thinking and academic performance. Educational Psychology, 22(3), 331-348. Recuperado de http://hd1.hadle.net/10722/43528. doi: 10.1080/01443410220138557

Zhang, L. F. (2004). Revisiting the predictive power of thinking styles for academic performance. The Journal of Psychology: Interdisciplinary and Applied, 138(4), 351-370. doi: 10.3200/JRLP.138.4.351-370

Zhang, L. F. (2006). Thinking styles and the big five personality traits revisited. Personality and Individual Differences, 40(6), 1177-1187.

Zhang, L. F., \& Sternberg, R. J. (2005). A threefold model of intellectual styles. Educational Psychology Review, 17(1), 1-53. doi: 10.1007/ s10648-005-1635-4

\section{Sobre os autores}

Cristiano Mauro Assis Gomes é Doutor em Educação e Coordenador do Laboratório de Investigação da Arquitetura Cognitiva da Universidade Federal de Minas Gerais.

Eunaihara Ligia Lira Marques é Mestre em Psicologia pela Universidade Federal de Minas Gerais e Docente na Faculdade de Pará de Minas. 